\title{
Engineering REST-specific synthetic PUF proteins to control neuronal gene expression: a combined experimental and computational study
}

Stefania Criscuolo ${ }^{1 \$ \pi}$, Mahad Gatti lou ${ }^{1 \$ \S}$, Assunta Merolla ${ }^{1,2 \$}$, Luca Maragliano ${ }^{1,3 *}$, Fabrizia Cesca $^{1,4 *}$ and Fabio Benfenati ${ }^{1,3 *}$

${ }^{1}$ Center for Synaptic Neuroscience and Technology, Istituto Italiano di Tecnologia, Genova, 16132 Italy

${ }^{2}$ University of Genova, 16132 Italy

${ }^{3}$ IRCCS Ospedale Policlinico San Martino, Genova, Italy

${ }^{4}$ Department of Life Sciences, University of Trieste, 34127 Italy

* To whom correspondence should be addressed: Fabio Benfenati, Tel: +39-01071781434; Fax: +39-0105558491; Email: fabio.benfenati@iit.it. Correspondence may also be addressed to: Luca Maragliano, Tel: +39-0105558382; Fax: +39-0105558491; Email: luca.maragliano@iit.it; Fabrizia Cesca, Tel: +39-0405588727; Fax: +39-0105558491; Email: fabrizia.cesca@iit.it.

$\$$ The authors wish it to be known that, in their opinion, the first three authors should be regarded as joint First Authors

TPresent Address: Stefania Criscuolo, Systems and Synthetic Biology Laboratory, Telethon Institute of Genetics and Medicine (TIGEM), Pozzuoli (NA), 80078

§Present Address: Mahad Gatti lou, Division of Cancer Therapeutics, The Institute of Cancer Research (ICR), London, SW7 3RP, United Kingdom.

FPresent Address: Department of Life and Environmental Sciences, Marche Polytechnic University, Via Brecce Bianche, 60131, Ancona, Italy 


\section{SUPPLEMENTARY TABLE AND FIGURE LEGENDS}

Figure S1. REST 3'UTR RNA sequence (714 bp, corresponding to bp 3554-4266 of REST mRNA, NCBI \#NM_011263.2). RESTRNA8 is highlighted in red, RESTRNA16 spans the sequence of RESTRNA8 and flanking ribonucleotides highlighted in bold, marked by the black rectangle. The sequence of RESTRNA16-2.0 is in dark blue.

Figure S2. Characterization of the PUF16rest-2.0 construct. (A) PUF16rest-2.0 with its own target sequence RESTRNA16-2.0. Panel description as in Figure 1. (B) HEK293T cells were transfected with a plasmid coding PUF16rest-2.0, and protein expression was analyzed by western blotting using anti-Flag antibodies and antibodies for the housekeeping gene gapdh. (C) Confocal images of HEK293T cells transfected with the indicated construct were processed for indirect immunofluorescence using anti-Flag antibodies (red) to detect PUF constructs and Hoechst (blue) to visualize cell nuclei. The overlay image reveals the cytosolic localization of the construct. Scale bar: $10 \mu \mathrm{m}$. (D) Increasing concentrations of PUF16rest-2.0 were incubated with R16 and R2.0 sequences $(0-10 \mu \mathrm{M})$.

Figure S3. (A,B) Non-bonded interactions energies and distances for the R8-N1 interface in the three eight-repeat constructs. Even though no mutations were involved in this particular interface, the PUF8wt:RESTRNA8 system showed signs of stress in both analysis. (C-F) Distances and nonbonded interactions energies for the mutation sites R3-N14 (C,D), R4-N13 (E,F) and R14-N3 interfaces $(\mathrm{G}, \mathrm{H})$ in the three sixteen-repeat PUF proteins. Again, the PUF16wt:RESTRNA16 systems shows higher instability than the other two systems, particularly in the R4-N13 interface where a baseflipping event is maintained for the major part of the simulation.

Figure S4. Purification of PUF constructs. Cytosolic extracts of HEK293T cells transfected with the indicated constructs were incubated with magnetic beads conjugated to anti-Flag antibodies, eluted with $3 x$ Flag peptides, analyzed by SDS-PAGE on a $10 \%$ polyacrylamide gel and subsequently silverstained.

Figure S5. Evaluation of binding specificity of PUF8rest-ns and PUF16rest-ns. (A) EMSA analysis of PUF8rest-ns (0-1-2-5-10 $\mu \mathrm{M})$ incubated with biotinylated NRE (N) and RESTRNA8 (R8) ribonucleotide sequences. (B) PUF16rest-ns (0-1-2-5-10 $\mu \mathrm{M})$ was incubated with biotinylated 2XNRE (2N), and RESTRNA16 (R16). No specific RNA-protein complexes were detected, under any of the experimental conditions tested.

Figure S6. Crosslink RNA immunoprecipitation (CLIP) of the REST-PUF constructs mutated in the stacking residues for endogenous Gapdh mRNA. (A) CLIP was performed with agarose A beads, anti-Flag and IgG antibodies on N2a cell lysates transfected with the indicated constructs. Gapdh values were normalized against the input value and plotted as percent specific precipitation. IP values were not statistically significant vs IgG values, for all samples. Student's $t$-test, $p>0.05$. Data are shown as means \pm S.D. of $n=3$ independent experiments. (B) Immunoblot of immunoprecipitated complexes revealed with anti-flag antibodies. Input (INP) represents $20 \%$ of the cell extract before immunoprecipitation for all the indicated constructs. NC, negative control: cells transfected with the empty 'Flag' vector.

Figure S7. Modulation of the stability of REST mRNA through fusion of REST-specific PUF proteins and GLD2. (A) PUF-GLD2 fusion proteins are expressed in HEK293T cells. HEK293T were transfected with the indicated constructs (n.t: non transfected cells). Protein expression was analyzed by western blotting using anti-Flag antibodies and antibodies for the housekeeping protein $\beta$-tubulin. (B) PUF8wt-GLD2, but not PUF8rest-sY-GLD2, increases the stability of its target mRNA. HEK293T cells were co-transfected with the indicated constructs and the corresponding reporter plasmid (see Materials and Methods). Forty-eight hrs after transfection, luciferase activity was measured. The 
Renilla / Luciferase ratio was first calculated for each sample, and data (means \pm sem) were subsequently normalized to the activity of the non-transfected control ( $\mathrm{n}=3$ independent experiments). (C) PUF8rest-sY does not increase the stability of endogenous REST mRNA. N2a cells were transfected with the indicated constructs. After $48 \mathrm{~h}$, REST mRNA levels were quantified via qRTPCR analysis. Gapdh was used as control housekeeping gene; data were normalized to REST mRNA levels in cells transfected with the empty Flag vector, set to 1 . Data (means $\pm S D$ ) of $n=2$ independent experiments.

\section{Supplementary table 1: MicroRNA sites prediction analysis}

In order to evaluate the microRNA sites within mouse REST mRNA 3'UTR (NCBI \#NM_011263.2) we queried the microRNA database mirdb. The analysis predicted 66 miRNA sites. We analysed 42 microRNA prediction sites out of 66 according to the predicted score $>80$, which is considered by the algorithm the most likely to be real. From our study, none of 42 microRNA prediction sites overlapped with PUF target sequences in the REST mRNA.

\begin{tabular}{|c|c|c|c|c|c|}
\hline $\begin{array}{l}\text { Target } \\
\text { Detail }\end{array}$ & $\begin{array}{c}\text { Target } \\
\text { Rank }\end{array}$ & $\begin{array}{l}\text { Target } \\
\text { Score }\end{array}$ & miRNA Name & Gene Symbol & Gene Description \\
\hline Details & 1 & 99 & mmu-miR-8118 & Rest & RE1-silencing transcription factor \\
\hline Details & 2 & 98 & mmu-miR-495-3p & Rest & RE1-silencing transcription factor \\
\hline Details & 3 & 98 & mmu-miR-12200-5p & Rest & RE1-silencing transcription factor \\
\hline Details & 4 & 98 & mmu-miR-1192 & Rest & RE1-silencing transcription factor \\
\hline Details & 5 & 97 & mmu-miR-883b-3p & Rest & RE1-silencing transcription factor \\
\hline$\overline{\text { Details }}$ & 6 & 97 & mmu-miR-883a-3p & Rest & RE1-silencing transcription factor \\
\hline Details & 7 & 97 & mmu-miR-29b-3p & Rest & RE1-silencing transcription factor \\
\hline Details & 8 & 97 & mmu-miR-29c-3p & Rest & RE1-silencing transcription factor \\
\hline$\overline{\text { Details }}$ & 9 & 97 & mmu-miR-29a-3p & Rest & RE1-silencing transcription factor \\
\hline Details & 10 & 96 & mmu-miR-217-5p & Rest & RE1-silencing transcription factor \\
\hline Details & 11 & 95 & mmu-miR-677-3p & Rest & RE1-silencing transcription factor \\
\hline Details & 12 & 92 & mmu-miR-20a-5p & Rest & RE1-silencing transcription factor \\
\hline$\overline{\text { Details }}$ & 13 & 92 & mmu-miR-6383 & Rest & RE1-silencing transcription factor \\
\hline$\overline{\text { Details }}$ & 14 & 92 & mmu-miR-106b-5p & Rest & RE1-silencing transcription factor \\
\hline Details & 15 & 92 & mmu-miR-106a-5p & Rest & RE1-silencing transcription factor \\
\hline Details & 16 & 92 & mmu-miR-93-5p & Rest & RE1-silencing transcription factor \\
\hline Details & 17 & 92 & mmu-miR-20b-5p & Rest & RE1-silencing transcription factor \\
\hline$\overline{\text { Details }}$ & 18 & 92 & mmu-miR-17-5p & Rest & RE1-silencing transcription factor \\
\hline Details & 19 & 91 & mmu-miR-19b-3p & Rest & RE1-silencing transcription factor \\
\hline Details & 20 & 91 & mmu-miR-19a-3p & Rest & RE1-silencing transcription factor \\
\hline$\overline{\text { Details }}$ & 21 & 90 & mmu-miR-291a-3p & Rest & RE1-silencing transcription factor \\
\hline Details & 22 & 90 & mmu-miR-7001-3p & Rest & RE1-silencing transcription factor \\
\hline Details & 23 & 90 & mmu-miR-302d-3p & Rest & RE1-silencing transcription factor \\
\hline Details & 24 & 90 & mmu-miR-294-3p & Rest & RE1-silencing transcription factor \\
\hline$\overline{\text { Details }}$ & 25 & 90 & mmu-miR-669j & Rest & RE1-silencing transcription factor \\
\hline Details & 26 & 90 & mmu-miR-669i & Rest & RE1-silencing transcription factor \\
\hline Details & 27 & 90 & mmu-miR-295-3p & Rest & RE1-silencing transcription factor \\
\hline Details & 28 & 90 & mmu-miR-302b-3p & Rest & RE1-silencing transcription factor \\
\hline Details & 29 & 90 & mmu-miR-302a-3p & Rest & RE1-silencing transcription factor \\
\hline Details & 30 & 89 & mmu-miR-26b-5p & Rest & RE1-silencing transcription factor \\
\hline Details & 31 & 89 & mmu-miR-7649-3p & Rest & RE1-silencing transcription factor \\
\hline Details & 32 & 89 & mmu-miR-3473c & Rest & RE1-silencing transcription factor \\
\hline
\end{tabular}




\begin{tabular}{|c|c|c|c|c|c|}
\hline Details & 33 & 89 & mmu-miR-26a-5p & Rest & RE1-silencing transcription factor \\
\hline Details & 34 & 88 & mmu-miR-448-3p & Rest & RE1-silencing transcription factor \\
\hline Details & 35 & 87 & mmu-miR-6397 & Rest & RE1-silencing transcription factor \\
\hline Details & 36 & 86 & mmu-miR-5619-3p & Rest & RE1-silencing transcription factor \\
\hline Details & 37 & 85 & mmu-miR-6896-3p & Rest & RE1-silencing transcription factor \\
\hline Details & 38 & 84 & mmu-miR-153-3p & Rest & RE1-silencing transcription factor \\
\hline Details & 39 & 83 & mmu-miR-186-5p & Rest & RE1-silencing transcription factor \\
\hline Details & 40 & 82 & mmu-miR-130a-5p & Rest & RE1-silencing transcription factor \\
\hline Details & 41 & 82 & mmu-miR-7119-3p & Rest & RE1-silencing transcription factor \\
\hline Details & 42 & 81 & mmu-miR-335-3p & Rest & RE1-silencing transcription factor \\
\hline Details & 43 & 79 & mmu-miR-6343 & Rest & RE1-silencing transcription factor \\
\hline Details & 44 & 77 & mmu-miR-6418-5p & Rest & RE1-silencing transcription factor \\
\hline$\overline{\text { Details }}$ & 45 & 75 & mmu-miR-6360 & Rest & RE1-silencing transcription factor \\
\hline Details & 46 & 74 & mmu-miR-32-3p & Rest & RE1-silencing transcription factor \\
\hline Details & 47 & 73 & mmu-miR-7062-3p & Rest & RE1-silencing transcription factor \\
\hline Details & 48 & 72 & mmu-miR-3108-5p & Rest & RE1-silencing transcription factor \\
\hline Details & 49 & 71 & mmu-miR-692 & Rest & RE1-silencing transcription factor \\
\hline Details & 50 & 70 & mmu-miR-491-3p & Rest & RE1-silencing transcription factor \\
\hline Details & 51 & 66 & mmu-miR-298-3p & Rest & RE1-silencing transcription factor \\
\hline Details & 52 & 64 & mmu-miR-7116-3p & Rest & RE1-silencing transcription factor \\
\hline Details & 53 & 61 & mmu-miR-9-3p & Rest & RE1-silencing transcription factor \\
\hline Details & 54 & 60 & mmu-miR-669h-3p & Rest & RE1-silencing transcription factor \\
\hline Details & 55 & 60 & mmu-miR-669k-3p & Rest & RE1-silencing transcription factor \\
\hline Details & 56 & 59 & mmu-miR-6951-3p & Rest & RE1-silencing transcription factor \\
\hline Details & 57 & 58 & mmu-miR-877-3p & Rest & RE1-silencing transcription factor \\
\hline Details & 58 & 53 & mmu-miR-7215-5p & Rest & RE1-silencing transcription factor \\
\hline Details & 59 & 53 & mmu-miR-130b-5p & Rest & RE1-silencing transcription factor \\
\hline Details & 60 & 53 & mmu-miR-7014-3p & Rest & RE1-silencing transcription factor \\
\hline Details & 61 & 52 & mmu-miR-139-3p & Rest & RE1-silencing transcription factor \\
\hline Details & 62 & 52 & mmu-miR-7a-2-3p & Rest & RE1-silencing transcription factor \\
\hline Details & 63 & 52 & mmu-miR-7b-3p & Rest & RE1-silencing transcription factor \\
\hline Details & 64 & 51 & mmu-miR-6986-3p & Rest & RE1-silencing transcription factor \\
\hline Details & 65 & 51 & mmu-miR-7050-3p & Rest & RE1-silencing transcription factor \\
\hline Details & 66 & 50 & mmu-miR-6371 & Rest & RE1-silencing transcription factor \\
\hline
\end{tabular}




\section{SI Materials and Methods}

Primers

\begin{tabular}{|c|c|}
\hline REST3'UTR & $\begin{array}{l}\text { Fw 5'-GCATAAATCTTAGCAAATCCTCGGGAG-3' } \\
\text { Rv 5'-GGCAGACAAGGCAAGTGGTGTG-3' }\end{array}$ \\
\hline Gapdh3'UTR & $\begin{array}{l}\text { Fw 5'-GAAACCCTGGACCACCCAC-3' } \\
\text { Rv 5'-GTGGGTGCAGCGAACTTTATTG-3' }\end{array}$ \\
\hline REST CDS & $\begin{array}{l}\text { Fw 5'-TTCACATTTATACGGGCGTTC-3' } \\
\text { Rv 5'-CCTGCAGCAAGTGCAACTAC-3' }\end{array}$ \\
\hline Gapdh CDS & $\begin{array}{l}\text { Fw 5'-AGGTCGGTGTGAACGGATTTG-3' } \\
\text { Rv 5'-TGTAGACCATGTAGTTGAGGTCA-3' }\end{array}$ \\
\hline PUF8rest-ns & $\begin{array}{l}\text { Fw 5'-CAAATTTGCAAatAATGTTGTGCAGAAGTGTGTTACTC-3' } \\
\text { Rv 5'-GAGTAACACACTTCTgCACAACATTatTTGCAAATTTG-3' }\end{array}$ \\
\hline PUF8rest-s & $\begin{array}{l}\text { Fw 5'-GCATAAATTTGCCAATtACGTGGTTCAAAAATGTG-3' } \\
\text { Rv 5'-CACATTTTTGAACCACGTAATTGGCAAATTTATGC-3' }\end{array}$ \\
\hline PUF8rest-sh & $\begin{array}{l}\text { Fw 5'- GCATAAATTTGCCAATCACGTGGTTCAAAAATGTG-3' } \\
\text { Rv 5'-CACATTTTTGAACCACGTgATTGGCAAATTTATGC-3' }\end{array}$ \\
\hline PUF16rest-ns & $\begin{array}{l}\text { Fw Rep3 5'- } \\
\text { GGCACTGCAAATGTATGGTAATCGTGTTATTCAGAAAGCCCTGG-3' } \\
\text { Rv Rep3 5'- } \\
\text { CCAGGGCTTTCTGAATAACACGATTACCATACATTTGCAGTGCC-3' } \\
\text { Fw Rep45'-GTGTGAAAGATCAGAATGGCTGTCATGTTGTGCAGAAATG- } \\
\text { 3' } \\
\text { Rv Rep4 5'-CATTTCTGCACAACATGACAGCCATTCTGATCTTTCACAC-3 } \\
\text { Fw Rep12 5'-CAAATTTGCAAatAATGTTGTGcAGAAGTGTGTTACTC-3' } \\
\text { Rv Rep12 5'-GAGTAACACACTTCTgCACAACATTatTTGCAAATTTG-3' } \\
\text { Fw Rep14 5'-GTATGGAAGCTATGTGATTCGTCATGTTCTGGAACATG-3' } \\
\text { Rv Rep14 5'-CATGTTCCAGAACATGACGAATCACATAGCTTCCATAC-3' }\end{array}$ \\
\hline PUF8Notl & Fw 5'-GCATAAATTTGCCAATAACGTGGTTCAAAAATGTG-3' \\
\hline PUF8BamHI & Rv 5'-CACATTTTTGAACCACGTTATTGGCAAATTTATGC-3' \\
\hline PUF16Notl & Fw 5'-CATAGCGGCCGCACCATGGGTCGTAGCCGTCTG-3' \\
\hline PUF16BamHI & Rv 5'-CATAGGATCCGCCCAGGTCCACGCCATTTTTC-3' \\
\hline
\end{tabular}




\begin{tabular}{|l|l|}
\hline GLD2-FLAG & $\begin{array}{l}\text { Fw 5'-CCAGGGATCCACCATGCCATCTCCACCTAC-3' } \\
\text { Rv 5'-CCAGGGATCCTTGAGATACATTTGATGATGCCATC-3' }\end{array}$ \\
\hline PUF(GLD2 $)$ & $\begin{array}{l}\text { Fw 5'-CATTGCGGCCGCACCATGGGTCGTAG-3' } \\
\text { Rv 5'- CATTTCTAGACCCGCCCAGGTCCACGCCATTTTTC-3' }\end{array}$ \\
\hline RESTRNA8 & $\begin{array}{l}\text { Sense 5'-TCGAGTTTATATAGC-3' } \\
\text { Antisense 5'-CGCCGCTATATAAA3- }\end{array}$ \\
\hline NRE & $\begin{array}{l}\text { Sense 5'-TCGAGTGTATATAGC-3' } \\
\text { Antisense 5'-CGCCGCTATATACAC-3' } \\
\text { RESTRNA16 }\end{array}$ \\
\hline 2XNRE & $\begin{array}{l}\text { Sense 5'-TCGAGTGCTTTATATAAATTAGC-3' } \\
\text { Antisense 5'-CGCCGTATATTATATACAACAC-3' }\end{array}$ \\
\hline
\end{tabular}




\section{Criscuolo, Gatti et al. Figure S1}

\section{REST mRNA 3'UTR}

\begin{tabular}{|rll|}
\hline 1 & CUGAGCCUCGGCAGAAGCACCGUGCAGACUUUGUGAGCAUGCAAUUUUAA & 50 \\
51 & UUUGUAGACAAACGCAAGCUUGCUUUAAUUAGUCUCCAAGGCUGAGUUUU & 100 \\
101 & CAGUAACAUUCUUUUUCUUAGGACUGUACAUCUAUUUAGUGUUUGUUGCA & 150 \\
151 & UAAAUCUUAGCAAAUCCUCGGGAGUUAAUGUAAGAGGACAGAUAUGUAAC & 200 \\
201 & UAGCUCGUGCAGGCAGGUGCAAGGAGAAGGGUAAGAUGGUGGAACACACC & 250 \\
251 & ACUUGCCUUGUCUGCCUACAACCUGUUGGGUUUUCUUUUCACGGUAGUUC & 300 \\
301 & CUAAUUUUUAGUUACUUGUUUAGAUCGAUAAAAAUUGGCUUAGUAAAUUA & 350 \\
351 & CUUGAAGAAUUUGCCUGCUUUAUAUAAAUUAAGUUAGCACUUUACAGUUU & 400 \\
401 & CUUUAGAGAUGAAAAAAAAGAGAUUUUAAUUGGAGAGAAAUUCUCAACAU & 450 \\
451 & UGGACAUUGUAUCUGUCCAGGUAAUUGCUUCCUAACUUGCUAUCAAUAUU & 500 \\
501 & UUGUGUUUAUAUGUUAAUCGUUAUAAAAAGUGAUUUUUGUUUUUUGGGUA & 550 \\
551 & UUUUUUAUUUUGGUGCUUUUCUGGCUUAAGAUGUUGCACAUGGUUCUUGU & 600 \\
601 & UUUUGUUUCUUUAACCUAUGCAGUUAAUCUCCCUUCCCCUGAAACAGCGU & 650 \\
651 & UGUGUUAAAUAGUAACACUAUACAGAUAUAUGCAUGGUUUUUUUUUUUGU & 700 \\
701 & UUGUUUGUUUGUU & 713 \\
\hline
\end{tabular}




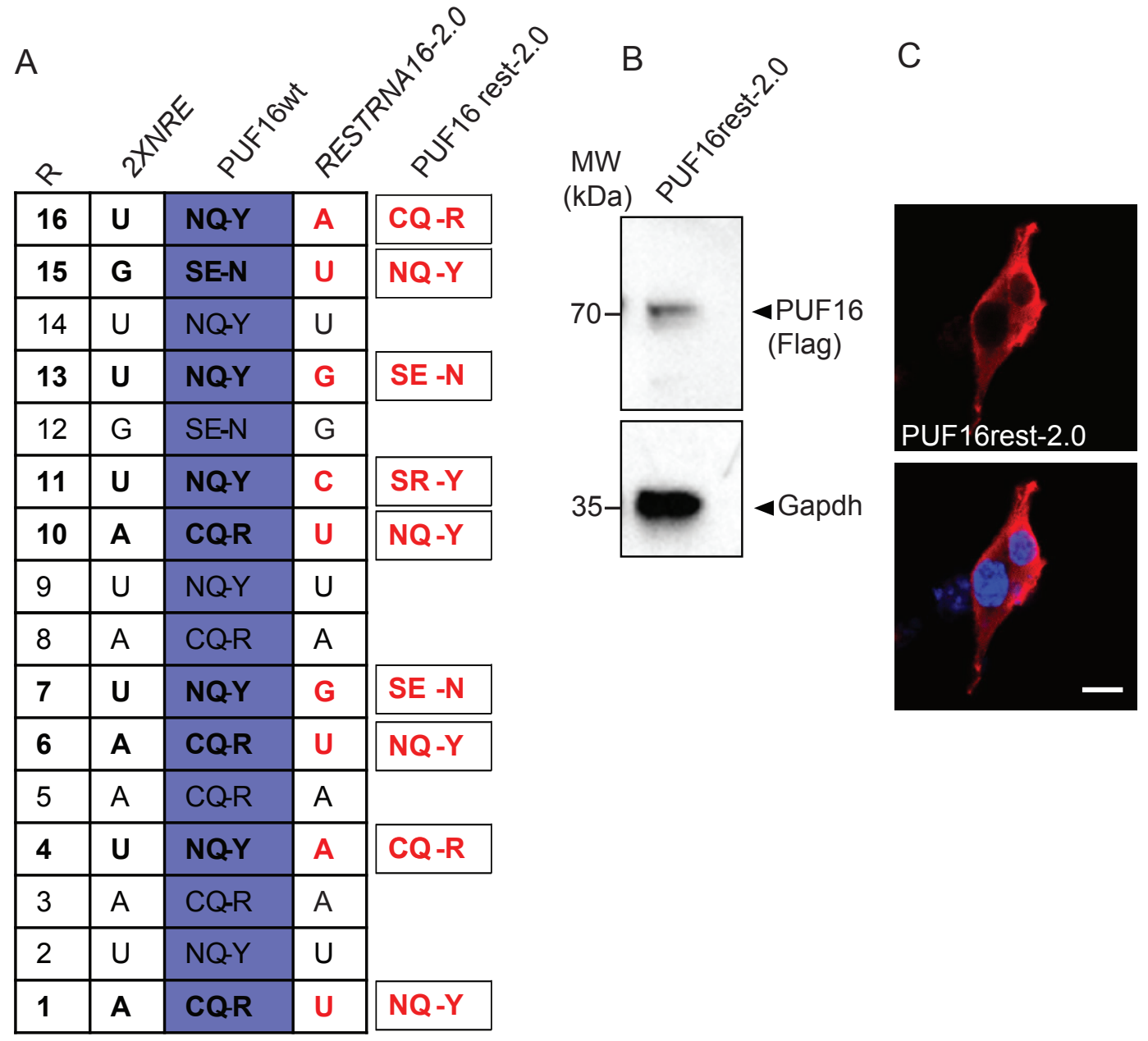

D PUF16rest-2.0 1-10 $\mu \mathrm{M}$

R16 R2.0 R16 R2.0 R16 R2.0 R16 R2.0 R16 R2.0

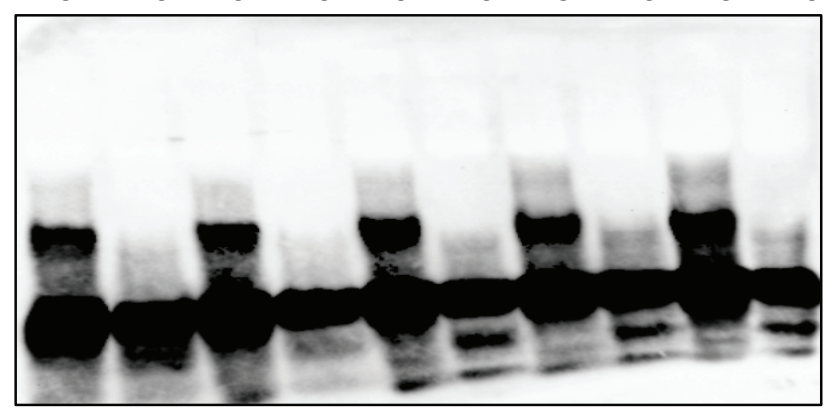


A

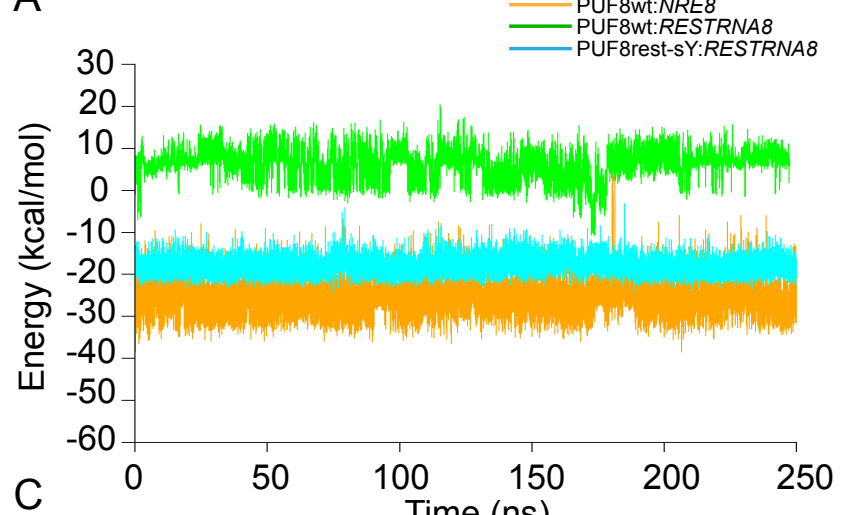

C

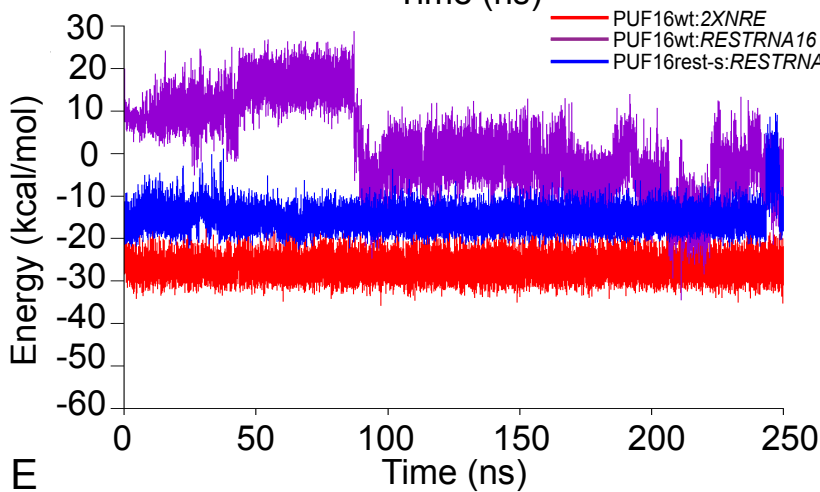

E

Time (ns)

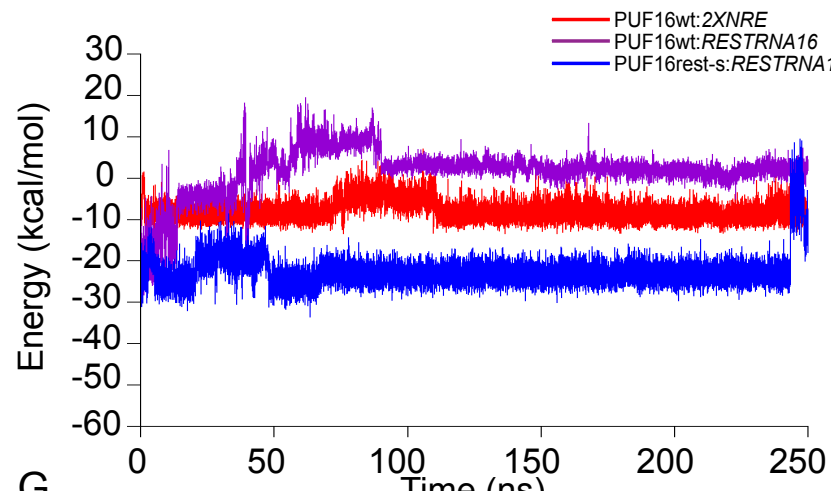

G

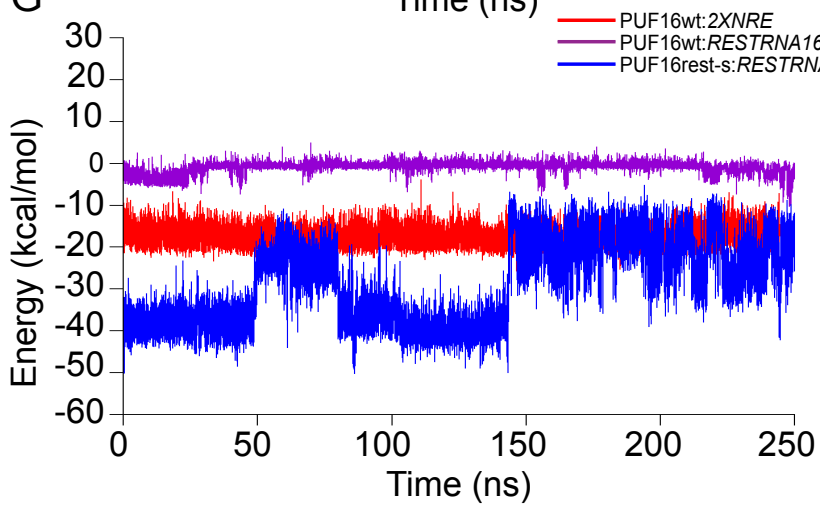

B
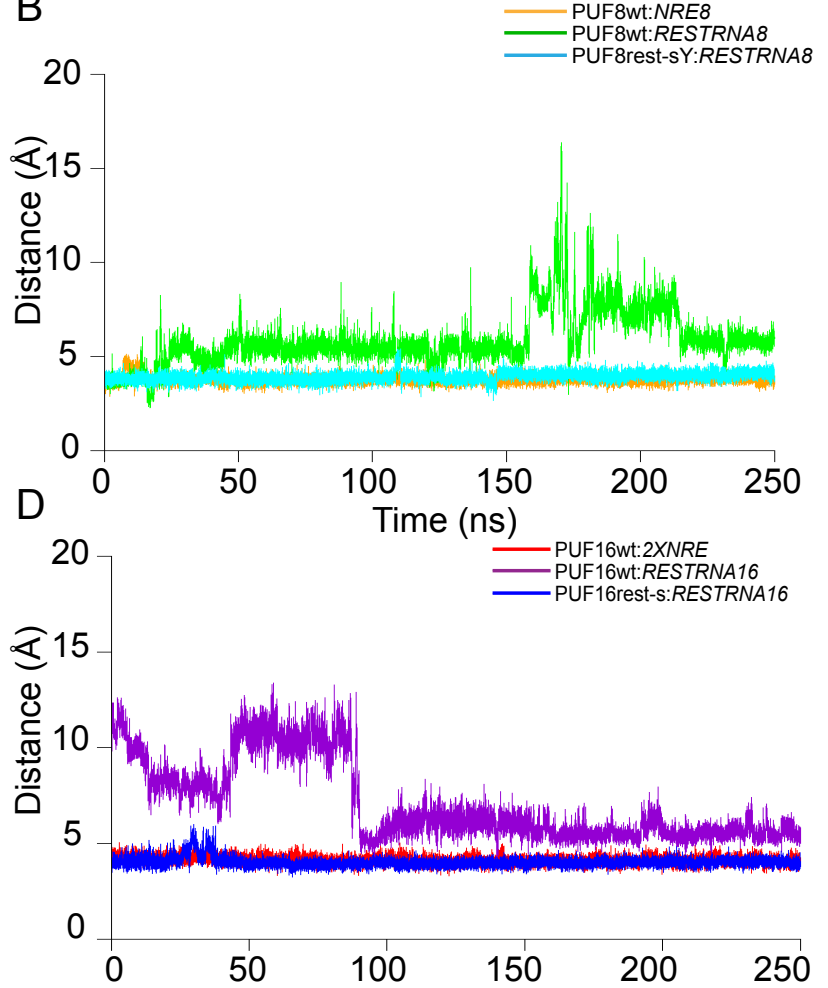

$\mathrm{F} \quad$ Time (ns)

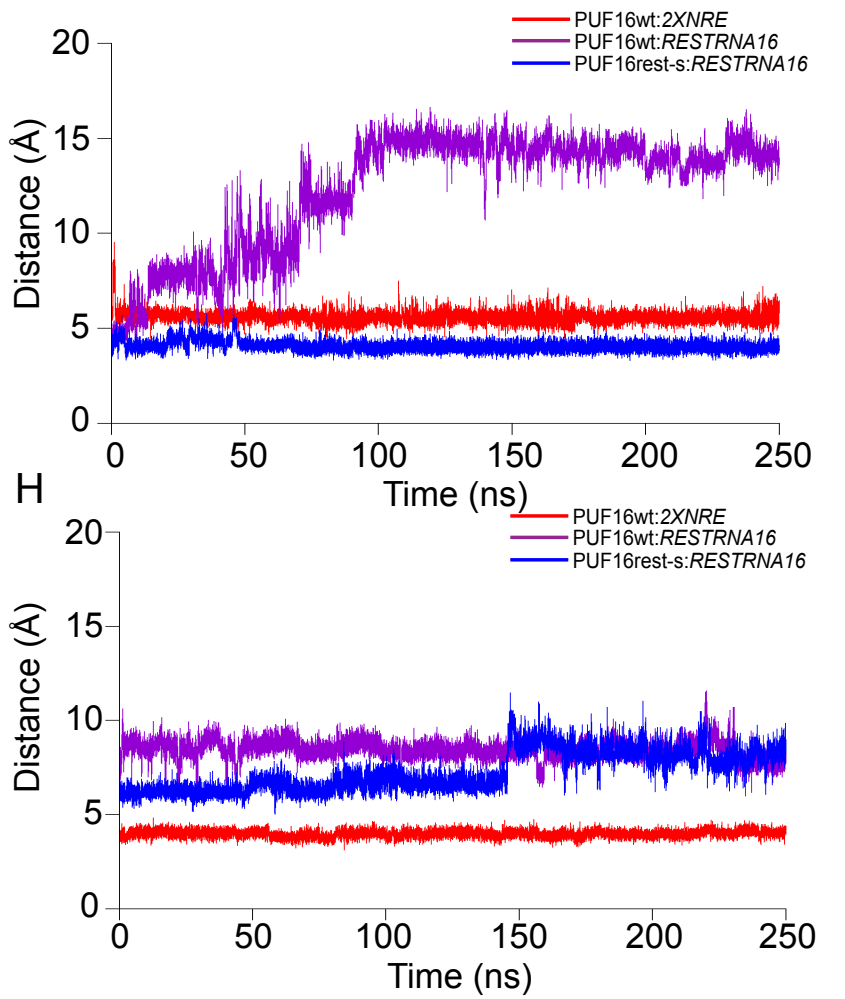


Criscuolo, Gatti et al. Figure \$4

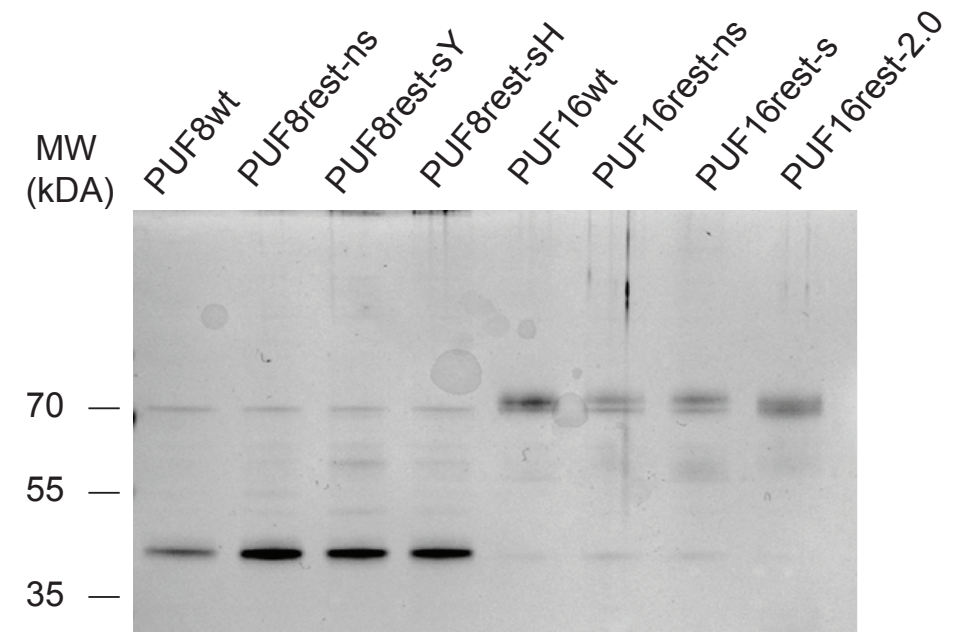


Criscuolo, Gatti et al. Figure S5

A

$$
\text { PUF8rest-ns } \quad 1-10 \mu \mathrm{M}
$$

$\begin{array}{llllllllll}\mathrm{N} & \mathrm{R} 8 & \mathrm{~N} & \mathrm{R} 8 & \mathrm{~N} & \mathrm{R} 8 & \mathrm{~N} & \mathrm{R} 8 & \mathrm{~N} & \mathrm{R} 8\end{array}$

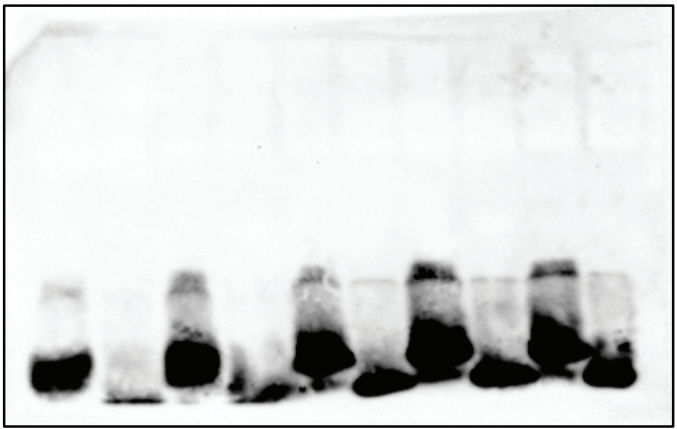

B

PUF16rest-ns $\quad 1-10 \mu \mathrm{M}$

2N R16 2N R16 2N R16 2N R16 2N R16

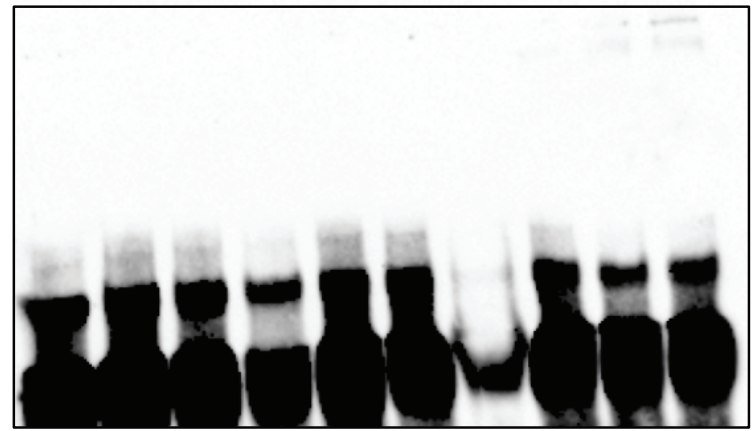


A
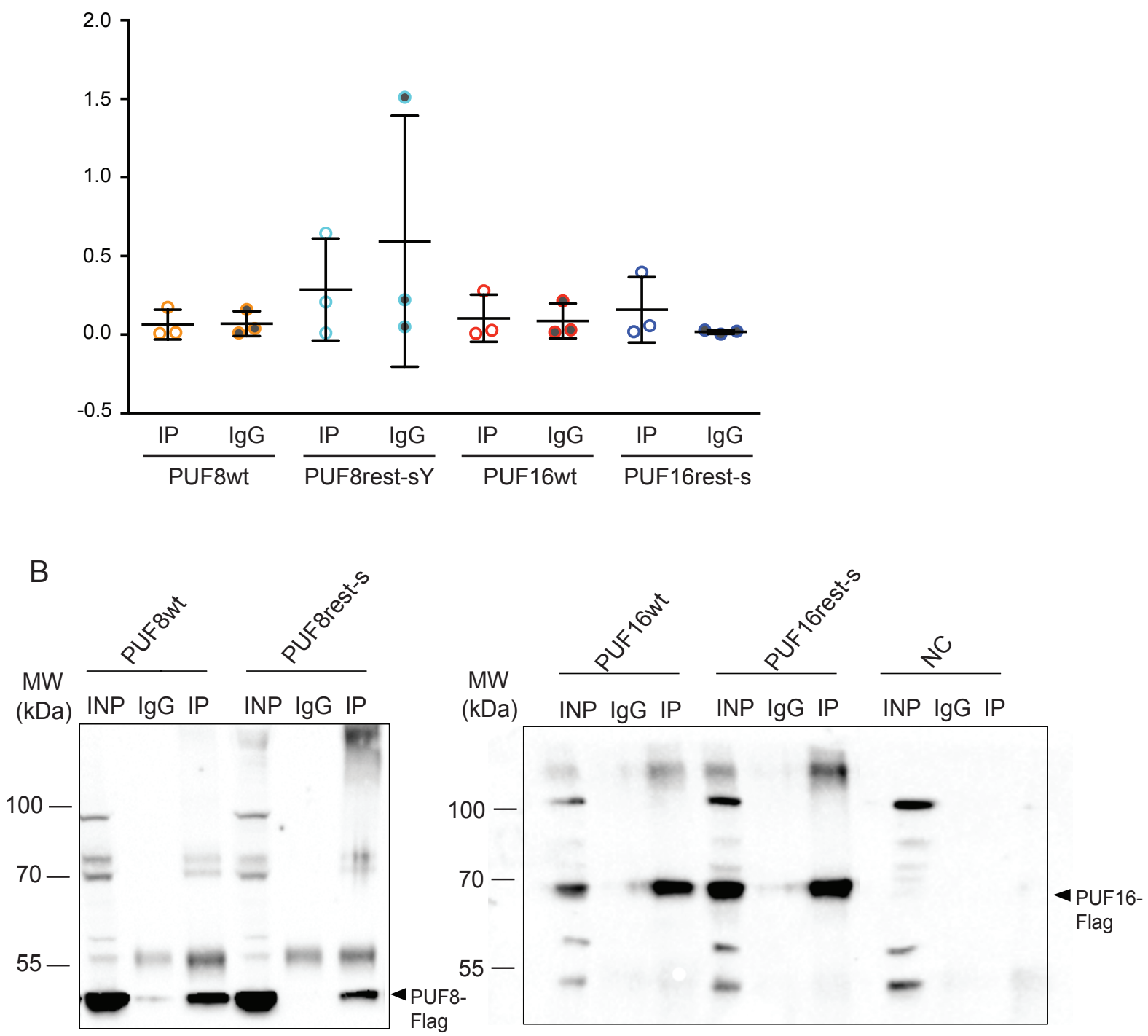
Criscuolo, Gatti et al. Figure S7

A

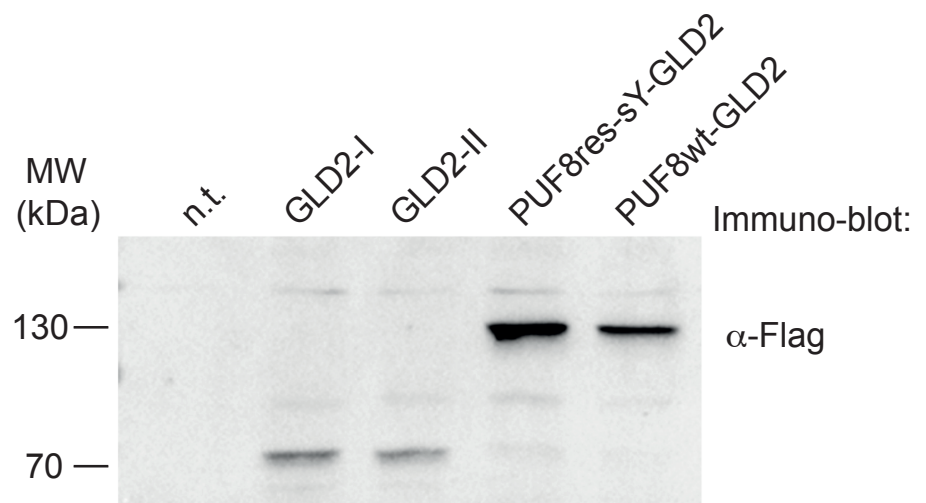

$55-$

$\alpha$-tubulin

B

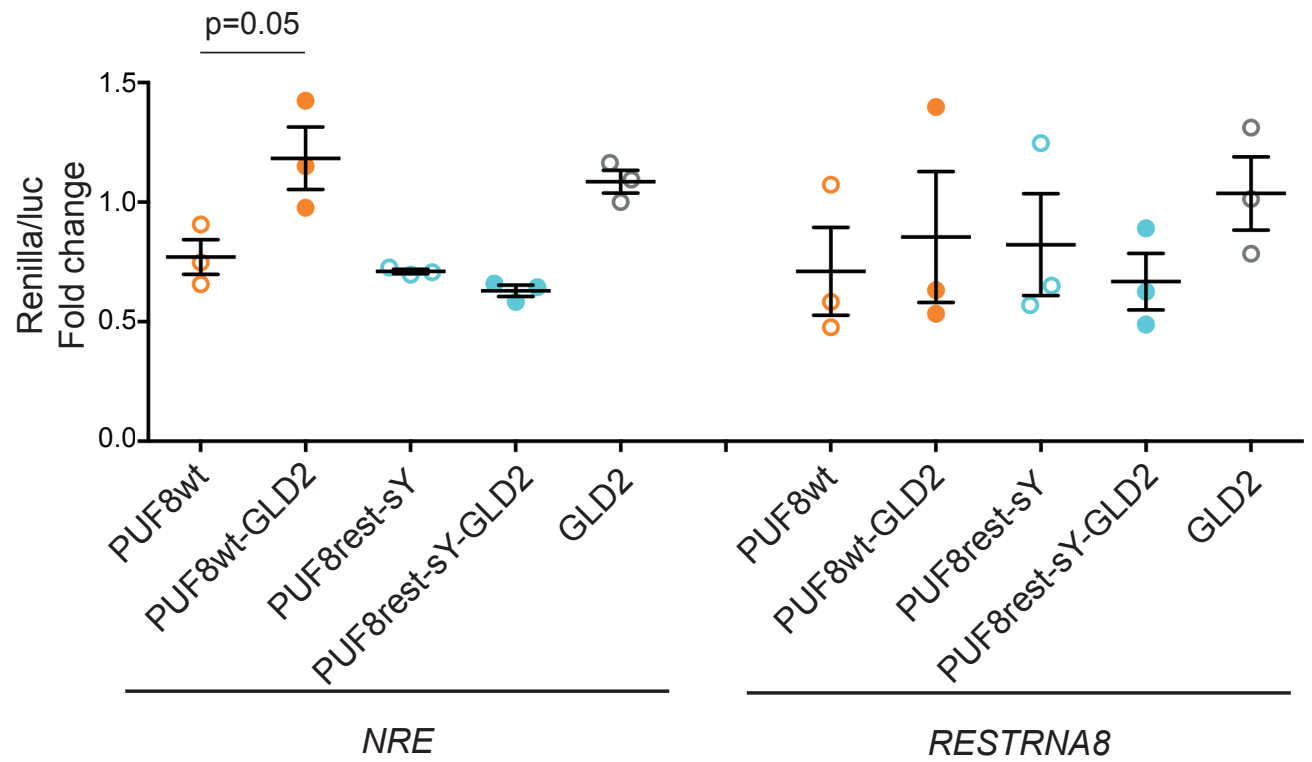

C

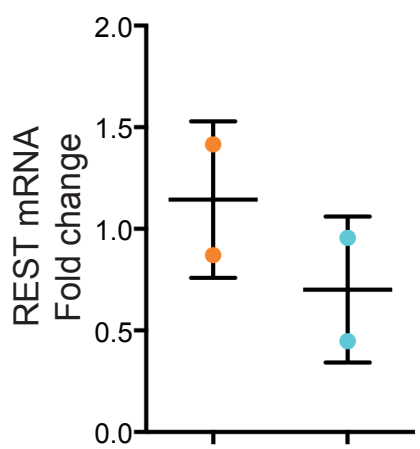

PUF8wt-GLD2

PUF8rest-sY-GLD2 